Matthew P. Canepa, Johnathan W. Hardy. "Persian palace architecture, garden design and digital archaeology. The role of 3D modeling in analyzing the palace of 'Emārat-e Kosrow at Qașr-e Širin, Iran"

\title{
Sébastien Gondet
}

\section{OpenEdition}

Édition électronique

URL : http://journals.openedition.org/abstractairanica/49788

DOI : 10.4000/abstractairanica.49788

ISBN : 1961-960X

ISSN : 1961-960X

Éditeur :

CNRS (UMR 7528 Mondes iraniens et indiens), Éditions de l'IFRI

Référence électronique

Sébastien Gondet, « Matthew P. Canepa, Johnathan W. Hardy. "Persian palace architecture, garden design and digital archaeology. The role of 3D modeling in analyzing the palace of 'Emārat-e Kosrow at Qașr-e Širin, Iran" », Abstracta Iranica [En ligne], Volume 40-41 | 2019, document 46, mis en ligne le 30 octobre 2019, consulté le 17 avril 2021. URL : http://journals.openedition.org/abstractairanica/ 49788 ; DOI : https://doi.org/10.4000/abstractairanica.49788

Ce document a été généré automatiquement le 17 avril 2021

Tous droits réservés 


\section{Matthew P. Canepa, Johnathan W. Hardy. "Persian palace architecture, garden design and digital archaeology. The role of 3D modeling in analyzing the palace of 'Emārat-e Kosrow at Qașr-e Širin, Iran"}

Sébastien Gondet

\section{RÉFÉRENCE}

Matthew P. Canepa, Johnathan W. Hardy. "Persian palace architecture, garden design and digital archaeology. The role of 3D modeling in analyzing the palace of 'Emārat-e Kosrow at Qașr-e Širin, Iran" in T. Daryaee (ed.). Sasanian Iran in the context of Late Anitquity. The Bahari lecture series at the Oxford University (= Ancient Iran Series 6). Irvine: Jordan Center for Persian Studies, 2018, p. 39-64

Le complexe de Qasr-e Shirin, situé à l'ouest de l'Iran dans la province de Kermanshah, a été très peu étudié jusqu'à présent. Situé non loin de la frontière avec l'Iraq, le site a souffert de nombreuses destructions durant la guerre Iraq-Iran auxquelles s'ajoutent celles liées à la pression de l'occupation moderne. Notons toutefois qu'en 1997 l'Iran a tenté de faire inscrire le site sur la liste du Patrimoine mondial de l'Unesco (https:// whc.unesco.org/en/tentativelists/889/) et qu'une équipe iranienne y a repris des recherches au cours des années 2000 [voir Abstracta Iranica 34-35-36 (2011-2013), R3.2.2. - $n^{\circ} 8$ ]. Les plans du palais, construit sur une vaste terrasse, celui du parc clôt attenant 
et de ses installations hydrauliques ainsi que les constructions voisines de Chahar Qapu (temple du feu ?) et d'une forteresse ne sont connus qu'à partir des relevés effectués à la fin du $19^{\mathrm{e}} \mathrm{s}$. par J. de Morgan puis ceux du début du $20^{\mathrm{e}} \mathrm{s}$. par G. Bell. Dans cet article, les A. s'appuient sur cette documentation pour reconstruire un modèle 3D du palais. Leur proposition de reconstruction se nourrit également d'observations effectuées sur des images satellitaires prises entre les années 1970 et aujourd'hui.

L'article s'ouvre sur une synthèse des données archéologiques et historiques disponibles concernant le site. On trouvera une discussion concernant la date de fondation du complexe qui, pour les A., est de la fin sassanide même s'il ne nie pas une réoccupation voire des reconstructions à dater du début de l'ère islamique. Leur démonstration s'appuie en grande partie sur des comparaisons avec d'autres constructions royales datant de la seconde moitié de la période sassanide. Après un bref exposé de leur méthodologie, les A. détaillent les choix effectués lors de la restitution du plan et des élévations pour chacun des secteurs du palais. On trouvera par exemple une discussion intéressante sur le style de l'iwan à l'entrée du palais. Les restitutions sont illustrées de nombreuses vues du modèle 3D produit ainsi que d'une nouvelle version des plans du site et du palais qui ont servi de base à ce travail.

3 S'il paraît effectivement important de réétudier ce site, les A. auraient pu toutefois profiter de cet article pour dresser des perspectives de recherche afin de compléter leur étude architecturale qui reste préliminaire. Leur restitution gagnerait en effet en qualité si elle se basait sur des observations des vestiges encore préservés in situ. Il est vrai qu'étant donné sa localisation, le site n'est encore aujourd'hui que très difficilement accessible. Toutefois une coopération plus étroite avec les équipes d'archéologues iraniens sur place et le recours aux technologies récentes de photogrammétrie, qui pourraient être mises en œuvre à partir de photographies ariennes ou de clichés pris au sol, permettraient de remédier à ce problème d'accès et enrichir la reconstruction de ce site.

\section{AUTEURS}

\section{SÉBASTIEN GONDET}

UMR 5133 CNRS-Université de Lyon 